\title{
Lactic acid bacteria antibacterial peptides: classification and current application
}

\author{
Kaiting Jiang ${ }^{1, *}$ \\ ${ }^{1}$ High school, Guangdong Experimental High School, Guangzhou, China
}

\begin{abstract}
Recently, the antibacterial peptides (bacteriocin) have spurred interest of scholars, and many studies on Lactic acid bacteria (LAB) and bacteriocin have been carried out. With the improvement of living standard and health awareness, people tend to pay more attention to food safety. Chemical preservatives are rejected because of their residual properties and toxicity. Applying antibacterial peptides, which serve as natural preservation, to food industry is an inevitable trend[1]. In this paper, the classification of LAB antimicrobial peptides and their application in food system are reviewed. The classification of bacteriocin, the current major food packaging technology, the application of LAB bacteriocin in different industries have been discussed.
\end{abstract}

\section{Introduction}

LAB are a group of Gram-positive bacteria, nonrespiring, non-spore-forming cocci or rods, which produce lactic acid as the major end-product of the fermentation of carbohydrates[2]. During the metabolic process of $\mathrm{LAB}$, it will generate many antibacterial substances, and most of them are acidic substance, antibacterial peptide, perhydrol etc. Bacteriocin refers to a kind of polypeptide or protein complex with antibacterial activity produced by ribosome during the common metabolic process[3]. LAB antibacterial peptide can inhibit the growth of pathogen in food effectively and safely[4]. Currently, LAB antibacterial peptides have spurred the interest of many researchers because of their potential of wide and effective application in food industry. The bacteriocin of LAB not only have the potential to be used as preservatives in food naturally but also are harmless and highly effective[5]. So far, there have been several successful examples of bacteriocin produced by LAB in the food industry. The most successful of them is Nisin. However, it still has problems, such as narrow bacteriostatic spectrum and no antibacterial effect to many Gramnegative bacteria, which hinders its further use in the food industry. Therefore, it is necessary to have further research on the LAB bacteriocin to solve these issues or look for alternatives to Nisin [6].

\section{The classification of lab bacteriocin}

The LAB bacteriocin is mainly divided into four categories [7].

The first class bacteriocin is Lantibiotic, which is a membrane active peptide with molecular weight less than $5 \mathrm{ku}$, and is a small molecule modified peptide containing 19-50 amino acid molecules. They have special amino acid, such as Lanthionine, $\beta$-methyllanthionine, Dehydroalanine, and Dehydrobutyrine. According to the ring-rigid structure that is formed by these special Amino acids, Lantibiotic can be classified into two sub-group, Class Ia and Class Ib. The Lantibiotic in Class Ia has a spiral shape with positive charge of 2-7. It has 21-38 amino acid residues, and its molecular mass are between 2164 to $3488 \mathrm{u}$. Moreover, it has tight rigidity and it is a peptide with a hydrophobic group. Nisin is a typical bacteriocin in Class Ia. Ib bacteriocin are spherical, negatively charged or neutral. Usually, the residues of amino acids in the molecules are less than 19, and the molecular mass is between 1954 and $2042 \mathrm{u}$.

The second class consists of non-lanthionin with a molecular weight of less than $10 \mathrm{ku}$ and polypeptides that have little or no modification. It has thermal stability, and can be classified into four sub-group. Class Ila is a kind of small molecular thermostable peptide with strong Listeria resistance activity, which is composed of S-S bridge. The N-terminal amino acid sequence is YGNGVXC. Class IIb is composed of two peptide oligomers with different amino acid sequences. Usually it needs 2 peptides to form together to be active. Class IIc is cyclic bacteriocin that are covalently connected by $\mathrm{N}$-terminal and C-terminal bonds. Class IId, nonpediococin, has not been modified after translation.

The third class is the macromolecular thermolabile peptide with a molecular weight greater than $30 \mathrm{ku}$. Most of this kind of bacteriocin are produced by Lactobacillus. It loses its activity at $100^{\circ} \mathrm{C}$ or lower temperature for 30 minutes. Helveticin V-1829, and acidophilucin A belongs to this class.

\footnotetext{
Corresponding author: KaitingJiang@126.com
} 
The fourth class is complex bacteriocin, is formed by hydrophobic bacteriocin, the macromolecule like carbohydrate and lipid group with positive charge [3].

\section{Application of lab as food pre- servation in food packaging system}

In order to reduce or eliminate the pathogen and spoilage in food, different techniques have been developed recently. The four main techniques are incorporation, nanotechnology, coating technology, and directly adding, which are elaborated below.

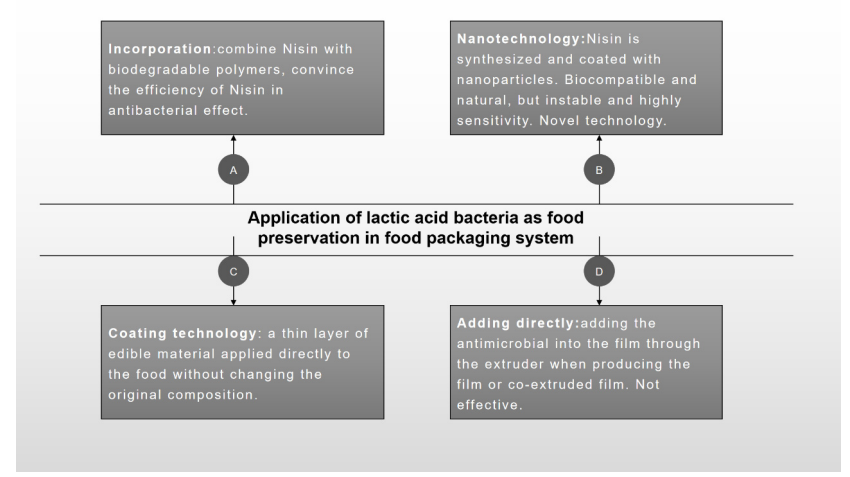

Figure 1. Application of LAB as food preservation in food packaging system

\subsection{Incorporation}

The first one is to combine Nisin with biodegradable polymers such as hydroxypropylmethycellulose, chitosan, sodium caseinate and polyactic acid, then they can migrate into the food through diffusion and partitioning[3]. It showed that Nisin could effectively maintain the antibacterial efficiency[9].

\subsection{Nanotechnology}

The second method is Nanotechnology, in which Nisin is synthesized and coated with nanoparticles such as nanoliposomes, nanoemulsions, nanoparticles and nanofibers. For the liposomes, some small artificial vesicles with sphere shape, Nisin is always incorporated into it in the food industry. The properties of small size, biocompatibility and naturalness allow it to have chance in obtaining approval in food-using. However, the properties of instability, highly sensitivity to environmental factors, such as temperature, variations in $\mathrm{pH}$, and its disruption when interacting with Nisin, also hinder it to be used effectively. For the nanoemulsions, nanoscale water-in-oil or oil-in-water disperses. They are nontoxic, nonirritant and more effective because they increase absorptive area, improve the taste and require little energy. In addition, they are thermodynamic stable. Nisin is always encapsulated into Nanoemulsion to improve the physical stability of agent. However, the drawbacks of this method, which are lack of functional, edible and permissible emulsifiers, also cannot be ignored. Nanoparticles are usually loaded with Nisin and are able to decrease the amount of Staphylococcus aureus and Listeria monocytogenes. For the nanofibers, they have high surface area-to-volume ratio, which increases their use in the food industry. The current package method is to embed it with antimicrobial agents, flavor-or odor-absorbent agents, oxygen scavengers, antioxidants, moisture-absorbent agents, and many other bioactive components[5].

\subsection{Coating technology}

The third method is the coating technique, in which a thin layer of edible material is applied directly to the food as a food package without changing the original composition[10]. Merve Duran and Mehmet Seckin Aday [11] used chitosan coatings as a carrier of Nisin to increase shelf life of fresh strawberry. As a result, they found that chitosan and Nisin can against aerobic mesophilic bacteria effectively.

\subsection{Adding directly}

The fourth method is adding the antimicrobial into the film through the extruder when producing the film or coextruded film. But in general, this approach is only marginally effective[12].

\section{The application of lab Bacteriocin in different industries}

\subsection{Application of LAB bacteriocin in meat}

The preservation of food using their natural and controlled microbial and/or their antimicrobial metabolites are referred to bio-protection[13]. Although the benefit of chemical preservation is considerable, with the increasing demand of natural food preservation, bacteriocin are being extensively studied. Moreover, because of their safety and innateness, they meet the demand of customers appropriately. The bacteriocin produced by LAB have already been used efficiently in raw meat preservation. Spraying the mixture of lactic acid and Nisin on the product has a good effect of inhibiting aerobic bacteria [14]. The previous studies in MRS broth and in a meat slurry showed that the inoculation of MRS broth with both Listeria monocytogenes $(4 \times 103 \mathrm{CFU} / \mathrm{mL})$ and Lb. curvatus CRL705 $(5 \times 107 \mathrm{CFU} / \mathrm{mL})$ led to a rapid decrease in the population of Listeria[13]. The research[15] has showed that the bacteriocin isolated from fish by-product can against certain germina such as Staphylococcus aureus, Salmonella sp., E. coli, Listeria monocytogenes etc.

\subsection{Application of lactic acid bacteriocin in dairy product}

The LAB bacteriocin have already been applied extensively in many fermentation dairy products. LAB and bacteriocin have been confirmed to have certain effect in bacteriostatic effect [16]. These results show the 
potential value of bacteriocin as food biological preservative in dairy production industry. Halil Ibrahim Kaya and Omer Simsek[17] added the pathogen-specific bacteriocin from LAB to the milk in a cocktail. As a result, it shows an inhibitory effect on B. cereus, $L$. monocytogenes and $S$. aureus. Nevertheless, the risk of using these bacteriocin are still exist. Bacteriocin isolated from dairy products can not only be added to dairy products to fight pathogens, but also serve as potential producers of antimicrobial proteins. LAB strains were found to be able to show antibacterial effect and inhibit undesirable microorganism in food[18]. However, the effect of antibiotic resistance of bacteriocin are still being considered. M.A. Herreros and H. Sandoval[19] have isolated several LAB strains from Armada cheese and examined whether it had antibacterial effect. However, none of them showed antibacterial activity against certain pathogen.

\subsection{Application of lactic acid bacteriocin in vegetables and fruits preservation}

Currently, the fresh-cut fruit and vegetable industry is searching for natural food preservation that can ensure the taste and safety of the food. In the experiment of Ana Allende, the Nisin-coagulin cocktail significantly inhibited growth of Listeria monocytogenes during the first three days after washing. Nevertheless, it could not prevent subsequent L. monocytogenes proliferation[20].

\section{Conclusion}

Bio-preservatives are natural and have no side effects[8]. LAB and their bacteriocin, especially Nisin, play important roles in dairy product, meat product and vegetables industry. However, it cannot be used extensively because of its instability and susceptibility to environment. In addition, superficial research on LAB bacteriocin except Nisin also lead to its inaccurate using. With more and more attention paid to bio-preservatives, the amount of research done on LAB and bacteriocins are increasing. The current anti-corrosion technology are mainly composed of coating technology, adding the agents directly, or embedding the bacteriocins into film of the food products[21]. With the development of technology and research, more and more novel strategies in bio-preservation are invented, such as nanotechnology, cheese-making transconjugants harbouring the Nisin production/immunity, paired-starter culture system which involved a Nisin-resistant Lactobacillus mesenteroides, and so on[22]. Since the vital potential of LAB and bacteriocin in food application and preservation, further study of the properties, including physical and chemical, structure-function relationships of bacteriocins are necessary. Moreover, further research on combining the bacteriocins according to their properties are needed, in order to achieve more efficient and effective food preservation. Finally, the influence on the taste and appearance of the product is also an important factor to be considered in the research process.

\section{Acknowlegdement}

The author is thankful to Dr. Cheng and Changling Wu for offering excellent teaching and directing of the article.

\section{References}

1.Woraprayote W, Malila Y, Sorapukdee S, et al. Bacteriocins from lactic acid bacteria and their applications in meat and meat products[J]. Meat ence, 2016, 120(oct.):118-132.

2.Sabatini, N., Chapter 24 - A Comparison of the Volatile Compounds, in Spanish-style, Greek-style and Castelvetrano-style Green Olives of the Nocellara del Belice Cultivar:: Alcohols, Aldehydes, Ketones, Esters and Acids, in Olives and Olive Oil in Health and Disease Prevention, V.R. Preedy and R.R. Watson, Editors. 2010, Academic Press: San Diego. p. 219-231.

3.Chenxi Zhang, Zhifei He, Hongjun Li. Research of bacteriocins from lactic acid bacteria and their applications in preservation ofmeat products [J]. Food and Fermentation Industries, 2017(07):276282.

4.Luya Ren, Yanyan Yang, Jianming Zhang, et al. Advance on antibacterial mechanism of lactic acid bacteria antimicrobial peptides $[\mathrm{J}]$. China Food Additives, 2015(1):143-149.

5.Khan, I. and D.-H. Oh, Integration of nisin into nanoparticles for application in foods. Innovative Food Science \& Emerging Technologies, 2016. 34: p. 376-384.

6.Chikindas, M.L., et al., Functions and emerging applications of bacteriocins. Curr Opin Biotechnol, 2018. 49: p. 23-28.

7.Cleveland $\mathrm{J}$, Montville $\mathrm{T} \mathrm{J}$, Nes I F, et al. Bacteriocins: safe, natural antimicrobials for food preservation[J]. International Journal of Food Microbiology, 2001, 71(1):1-20.

8.Santos J C P , Sousa R C S , Otoni C G, et al. Nisin and other antimicrobial peptides: Production, mechanisms of action, and application in active food packaging[J]. Innovative Food Science \& Emerging Technologies, 2018, 48:179-194.

9.Galus S , Kadzińska, Justyna. Food applications of emulsion-based edible films and coatings[J]. Trends in Food Science \& Technology, 2015, 45(2):273283.

10.Duran M , Aday M S , Zorba, Nükhet N. Demirel, et al. Potential of antimicrobial active packaging 'containing natamycin, nisin, pomegranate and grape seed extract in chitosan coating' to extend shelf life of fresh strawberry[J]. Food \& Bioproducts Processing, 2016:354-363.

11.Quintavalla S , Vicini L . Antimicrobial food packaging in meat industry[J]. Meat ence, 2002, 62(3):373-380. 
12.Castellano, P., et al., A review of bacteriocinogenic lactic acid bacteria used as bioprotective cultures in fresh meat produced in Argentina. Meat Sci, 2008. 79(3): p. 483-99.

13.Da Costa R J , Voloski, Flávia L. S, Mondadori R G, et al. Preservation of Meat Products with Bacteriocins Produced by Lactic Acid Bacteria Isolated from Meat[J]. Journal of Food Quality, 2019, 2019:1-12.

14.Marti-Quijal, F.J., et al., Fermentation in fish and byproducts processing: an overview of current research and future prospects. Current Opinion in Food Science, 2020. 31: p. 9-16.

15.Bingzheng $\mathrm{Xu}$, Yin Wang, Xiaoyue Liang, et al. Progress of Application Research on Lactic Acid Bacteria Bacteriocin [J]. Journal of Heilongjiang August First Land Reclamation University, 2015(1):60-63.

16.Kaya, H.I. and O. Simsek, Characterization of pathogen-specific bacteriocins from lactic acid bacteria and their application within cocktail against pathogens in milk. Lwt, 2019. 115.

17.García-Cano, I., et al., Lactic acid bacteria isolated from dairy products as potential producers of lipolytic, proteolytic and antibacterial proteins. Applied Microbiology and Biotechnology, 2019. 103(13): p. 5243-5257.

18.Herreros M A, Sandoval H, Gonzalez L, et al. Antimicrobial activity and antibiotic resistance of lactic acid bacteria isolated from Armada cheese (a Spanish goats' milk cheese)[J]. Food Microbiology, 2005, 22(5):455-459.

19.Allende A, Martinez B, Selma V, et al. Growth and bacteriocin production by lactic acid bacteria in vegetable broth and their effectiveness at reducing Listeria monocytogenes in vitro and in fresh-cut lettuce[J]. Food Microbiology, 2007, 24(7-8):759766.

20.Xiaoning Zhang, Yina Shang, Jing Chen, et al. Mechanism of Bacteriocins from Lactobacillus and Their Applications in Meat Products [J]. Food Research and Development, 2018(11).

21.Abee T, Krockel L, Hill C . Bacteriocins: modes of action and potentials in food preservation and control of food poisoning.[J]. International Journal of Food Microbiology, 1995, 28(2):169-185. 